\title{
Fiabilité et précision de la mesure des plis de peau en deux points symétriques de l'encolure lors de la tuberculination chez le zébu Gobra, Bos indicus Linnaeus, 1758 au Sénégal
}

\author{
Andrée Prisca Ndjoug NDOUR ${ }^{1 *}$, Fafa SOW ${ }^{2}$, Philippe Soumahoro KONE ${ }^{3}$ et \\ Ayayi Justin AKAKPO ${ }^{1}$
}

\author{
${ }^{1}$ Ecole Inter-Etats des Sciences et Médecine Vétérinaires (EISMV) de Dakar, Sénégal. \\ ${ }^{2}$ Centre de Recherches Zootechniques de Dahra-Djoloff, Sénégal; Km 4, Route de Linguère. \\ ${ }^{3}$ ECTAD - FAO, Boulevard du 30 Juin. BP: 16096 Kinshasa, République Démocratique du Congo. \\ *Auteur correspondant ; BP: 5077 Dakar-Fann, Sénégal ; E-mails: andreeprisca.ndour@gmail.com / \\ p.kone@hotmail.fr; Tel: +221775423124 (NDOUR)/+243829616580 (Kone).
}

\section{RESUME}

Au Sénégal, l'adhésion des éleveurs aux tests tuberculiniques est confrontée à la durée de leur réalisation au premier passage dans le troupeau. L'objectif de cette étude est d'évaluer si le fait de substituer la mesure non effectuée au Jour $0(\mathrm{~J} 0)$ une mesure en un point symétrique sur la face opposée de l'encolure au Jour 3 (J3) est envisageable. Ainsi, en octobre 2016, l'essai a été mené sur dix zébus Gobras de race pure (d'âge et de sexe différents) du Centre de Recherches Zoonotiques de Dahra Djoloff (Louga). Les mesures ont été prises et répétées dix fois sur le même animal, avec le même cutimètre, le même jour, par chacun des trois opérateurs. L'épaisseur moyenne du pli cutané était de 9,14 mm [2,09-25,62 mm (IC 95\%)] et 9,7 mm [3,67$15,92 \mathrm{~mm}$ (IC 95\%)] pour les faces droites et gauche respectivement. Suivant la méthode de Charrette et le procédé VARCOMP du logiciel SAS, la précision et la fiabilité étaient plus élevées pour la face gauche (reproductibilité de $90,14 \%$ et répétabilité de $86,66 \%$ ) que la face droite (reproductibilité de $86,86 \%$ et répétabilité de $82,46 \%$ ). Il a été montré dans cette étude qu'il est possible de remplacer la mesure de l'épaisseur cutanée à $\mathrm{J} 0$ par une autre effectuée au point symétrique du lieu de la tuberculination à J3, chez le bovin Gobra. (C) 2019 International Formulae Group. All rights reserved

Mots clés : Zébu-Gobra, Mesure Plis de peau, Reproductibilité-Répétabilité, Tuberculination, Sénégal.

\section{Reliability and Accuracy of skin fold measurement in two symmetrical points of the neck during in zebu Gobra, Bos indicus Linnaeus, 1758 in Senegal}

\begin{abstract}
In Senegal, the participation of breeders to tuberculin test is hampered by the duration of the first passage in the herd. The objective of this study is to evaluate whether the substitution of the measurement not performed on Day 0 (D0) by a measurement at a symmetrical point on the opposite side of the neck on Day 3 (D3) is possible. Thus, in October 2016, a trial was carried out on ten zebu Gobra of pure breed (of different sex and age) of the Centre de Recherches Zoonotiques de Dahra Djoloff (Louga, Senegal). Measures were taken and repeated ten times on the same animal, with the same calliper, the same day, by each of three
\end{abstract}


operators. The average thickness of skinfold is $9.14 \mathrm{~mm}$ [2.09-25.62 mm (IC 95\%)] and $9.7 \mathrm{~mm}$ [3.67-15.92 $\mathrm{mm}$ (IC 95\%)] for right and left sides respectively. According to the method of Charrette and VARCOMP procedure under SAS software, the accuracy and reliability are higher for left side (reproducibility of $90.14 \%$ and repeatability of $86.66 \%$ ) than right side (reproducibility of $86.86 \%$ and repeatability of $82.46 \%$ ). This study shows that it is possible to replace the measure of skin thickness at D0 by another one at a symmetrical point of tuberculin inoculation point at D3 for Gobra cattle.

(C) 2019 International Formulae Group. All rights reserved

Keywords: Zebu Gobra; Measure; Skinfold; Repeatability-Reproducibility, Senegal.

\section{INTRODUCTION}

La tuberculose bovine due à Mycobacterium bovis (TbB) est une pathologie infectieuse chronique, débilitante observée chez les mammifères domestiques (bovins, dromadaires, etc.) et sauvages (buffle d'Afrique, antilope, cerf, etc.) y compris l'homme. Cette zoonose est un véritable problème de santé publique, qui et en 2016, a occasionné 147000 nouveaux cas et 12500 décès chez l'homme dans le monde (OMS et al., 2017). Le poids économique réel de la tuberculose zoonotique en santé publique et sur les échanges commerciaux à l'échelle internationale est inconnu ; cependant, selon certains auteurs, il peut être estimé à plusieurs millions de dollars US (Boukary et al., 2011 ; OIE, 2012 ; Kwaghe et al, 2015).

Dans les pays développés, en raison de son impact en santé publique et de la nature non stérilisante du traitement chez l'animal, la stratégie "test-abattage" c'est-à-dire abattage de tout animal répondant positivement à la tuberculination, a permis de réduire l'incidence voire d'éliminer la tuberculose bovine en France, au Royaume Uni, aux Etats Unis, en Ireland, etc. (de la Rua-Domenech et al., 2006 ; Schiller et al., 2010 ; Alvarez et al., 2012 ; de Garine-Witchatitsky et al., 2013). En Afrique, par contre, la TbB est encore endémique et dans plus d'une quarantaine de pays africains à l'exception du Botswana et du Zimbabwe où elle n'a jamais été observée chez les animaux tant domestiques que sauvages, (Amanfu, 2006; Zinsstag et al., 2006; Garine-Witchatitsky et al., 2013). Selon la région éco-géographique et le système d'élevage considérés, les prévalences de la $\mathrm{TbB}$ varient de $0,1 \%$ à $48 \%$ chez les bovins et; de 0,6 à 24,4\% chez l'homme (Boukary et al., 2011 ; Vikou et al., 2018).

Au Sénégal, la TbB a été décrite depuis les années 1930. Malgré le risque pour la santé humaine et des prévalences non négligeables $(8,7 \%$ en 2015$)$, la $\mathrm{TbB}$ ne fait l'objet que d'une déclaration obligatoire (décret $\mathrm{n}^{\circ}$ 2002-1094 du 4 novembre 2002 relatif à la Police Sanitaire des animaux) mais ne figure toujours pas sur la liste des maladies animales prioritaires au Sénégal (Diagne, 2009 ; Sénégal, 2015). Or, conformément aux objectifs de développement durable des Nations Unies adoptés en 2015 par l'OMS, la TbB doit être éradiquée d'ici 2030-2035. Les tests d'hypersensibilité retardée ou tuberculination sur l'animal vivant ne sont pas, pour le moment, réalisés mais ils sont désormais prévus pour les troupeaux laitiers.

$\mathrm{La}$ tuberculination par la voie intradermique est le test de référence de l'OIE (2012) pour déceler l'infection tuberculeuse chez les animaux vivants. Le principe du test repose sur la mise en évidence d'une réaction inflammatoire locale apparaissant chez un sujet déjà sensibilisé par la présence de la bactérie (Figure 1). La technique de l'intradermoréaction (IDR), peut être simple (utilisation de la tuberculine bovine uniquement) ou comparative (utilisation de tuberculines bovine et aviaire en deux points différents de l'encolure). L'administration de la tuberculine se fait soit au milieu du tiers de l'encolure (site réglementaire en Europe et en Afrique conformément aux recommandations de l'OIE) soit à la base de la queue (site réglementaire aux Etats Unis). Sa réalisation consiste à délimiter une zone où les poils sont rasés au niveau du tiers moyen de l'encolure, d'effectuer une première mesure de 
l'épaisseur du pli de peau (mesure à J0) avant l'injection de la tuberculine (par la voie intradermique) puis une deuxième mesure, soixante-douze heures après (mesure à $\mathrm{J} 3$ ). Les résultats sont alors interprétés, à la fois, de manière qualitative et quantitative. Au plan qualificatif, l'apparition d'une réaction inflammatoire ou de douleur des conduits lymphatiques de la zone ou des nœuds lymphatiques, d'exsudation, d'œdème diffus ou étendu, de nécrose au point d'injection sont caractéristiques d'une suspicion d'une réaction positive. En fonction de la différence entre les deux mesures, la réaction est qualifiée de négative si elle est inférieure à 2 $\mathrm{mm}(0<\mathrm{J} 3-\mathrm{J} 0<2 \mathrm{~mm})$; de douteuse si comprise entre 2 et $4 \mathrm{~mm}(2 \mathrm{~mm}<\mathrm{J} 3-\mathrm{J} 0<$ $4 \mathrm{~mm}$ ); de positive si supérieure ou égale à 4 $\mathrm{mm}(\mathrm{J} 3-\mathrm{J} 0 \geq 4 \mathrm{~mm})$. Les tests tuberculiniques sont donc une démarche lourde nécessitant la contention des animaux, deux passages en trois jours dans les élevages, des moyens logistiques et financiers assez conséquents.

Si les programmes officiels de contrôle de la TbB sont quasi inexistants en Afrique Subsaharienne, c'est en partie en raison des contraintes financières et du mode d'élevage (pâture, nomadisme et transhumance). Au Sénégal, la majorité des animaux est chaque jour conduite au pâturage, ce qui ne favorise pas l'accès aux troupeaux deux fois en soixante-douze heures pour la mise en œuvre de la tuberculination. Cette pratique requiert l'immobilisation des troupeaux par les éleveurs; or, ces derniers considèrent cette obligation comme une entrave à l'exercice de leur profession et une atteinte à leur liberté. De même, le strict respect des deux passages systématiques et le temps de réalisation de
l'IDR (2-10 minutes suivant l'expérience) ne sont donc pas favorables à l'adhésion des éleveurs à ce type de protocole. En parallèle à une première étude sur l'évaluation de la prévalence de la $\mathrm{TbB}$ (par tuberculination) au sein du cheptel bovin au Sénégal, une expérimentation relative à la mesure de l'épaisseur du pli cutané a été menée sur des zébus Gobra, Bos indicus, Linneaus 1758. Cette seconde étude a été envisagée suite au refus d'adhésion récurrent d'éleveurs à participer au protocole en raison de la longue durée de réalisation de l'IDR et; par conséquent, la baisse du nombre d'animaux par troupeaux à tester. La finalité de cette approche méthodologique au Sénégal est donc d'alléger les contraintes liées à l'immobilisation et la durée de l'IDR. Le but de cette méthode alternative est d'évaluer la possibilité de remplacer la mesure à J0 (avant l'injection de la tuberculine) par une autre, en un point symétrique sur la face opposée de l'encolure en cas d'IDR simple (IDS) ou comparative (IDC), le jour de la lecture du résultat (soixante-douze heures après administration de la tuberculine), tel que recommandé par l'OIE (2012). Cette démarche méthodologique consiste alors à réaliser les mesures $\mathrm{J} 0$ et $\mathrm{J} 3 \mathrm{chez}$ les animaux présentant une réaction inflammatoire lors du deuxième passage dans le troupeau. Au cours de cette étude, il s'agit de comparer l'épaisseur du pli de peau sur les faces droite et gauche de l'encolure du zébu Gobra pour s'assurer qu'il n'y a pas de différence significative d'épaisseur cutanée et; ainsi, apprécier les niveaux de fiabilité et de précision des mesures effectuées. 

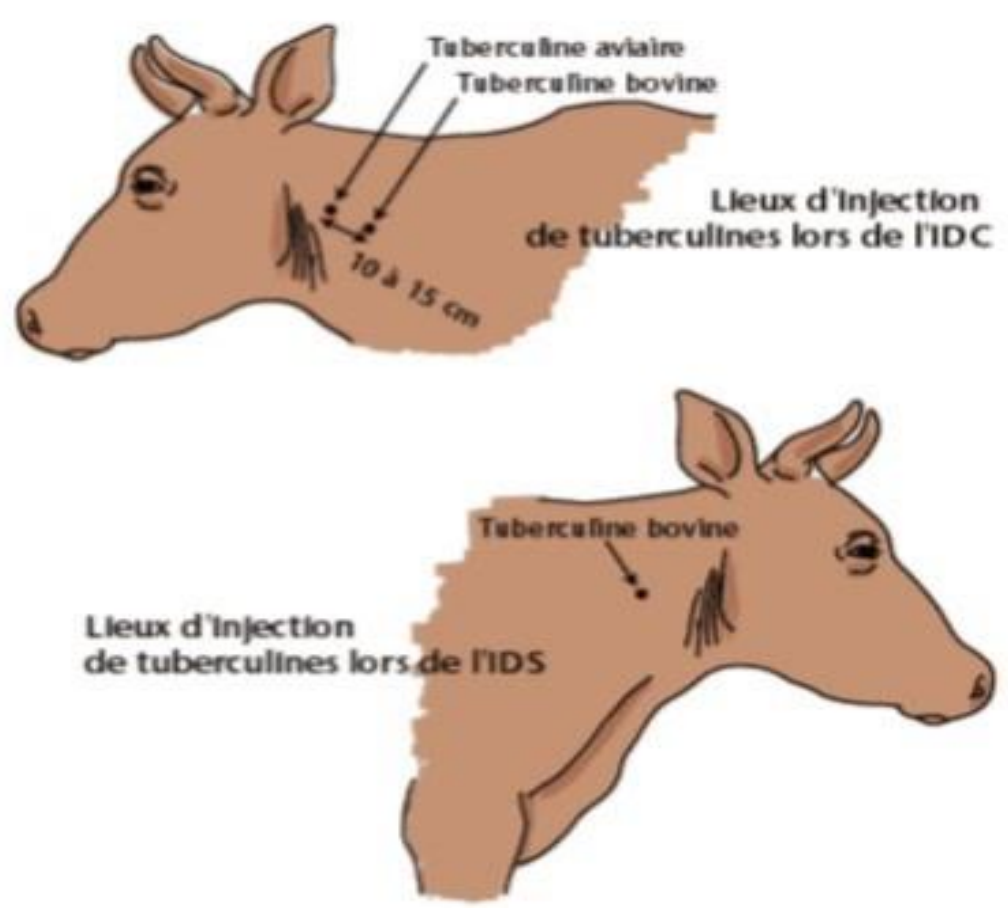

Figure 1 : Lieux d'injection lors de la tuberculination (http://www.gds64.fr).

\section{MATERIEL ET METHODES}

Cette expérimentation a été réalisée en octobre 2016 au Centre de Recherches Zootechniques (CRZ) de Dahra Djoloff dans la région de Louga, Sénégal (Figure 2).

\section{Matériel}

Le matériel vivant est constitué par des zébus mâles et femelles de race Gobra du CRZ de Dahra. Le zébu Gobra, largement répandu au Sénégal est une race apte à la production, à la fois, de viande et de lait.

Les mesures du pli cutané ont été prises avec un cutimètre (pied à coulisse) électronique de marque Pearl série PE 7811. Chacun des opérateurs disposait d'une fiche sur laquelle l'âge, le sexe ainsi que les différentes mesures ont été reportées.

\section{Méthodes}

Dix (10) zébus Gobra de différentes catégories d'âges (adultes, juvéniles, veaux) ont été tirés au hasard de la base de données transmise par le CRZ: (04) adultes, quatre (04) jeunes et deux (02) veaux.
Le choix du CRZ de Dahra a été orienté par le fait qu'il soit l'un des rares centres de recherche au Sénégal disposant d'un noyau de race pure de Zébu Gobra. Le critère "race pure", a été retenu dans le but d'obtenir une certaine homogénéité dans l'épaisseur du pli de peau des animaux de cette espèce.

En vue de l'appréciation de la précision et de la fiabilité des mesures des plis cutanés, deux zones symétriques au milieu du tiers moyen de l'encolure (Figure 1) ont été délimitées sur chacune des faces comme pour la réalisation de la tuberculination. Au niveau de ces zones, le poil a été rasé avec soin de sorte à ne pas occasionner de lésions au niveau de la peau ni de réaction inflammatoire non spécifique (usage de ciseaux courbes de préférence) qui pourrait induire des confusions à la lecture du résultat de la tuberculination.

Sur chaque animal, le pli de peau a été mesuré à dix (10) reprises par le même opérateur avec le même instrument, le même jour. 
La prise des mesures a été réalisée par trois (03) agents techniques vétérinaires volontaires du CRZ, tous droitiers, qui ont été préalablement formés à l'utilisation du cutimètre et à la mesure du pli de peau chez les bovins.

\section{Calculs statistiques}

Les données sur le sexe et l'âge des bovins ont été saisies sur Excel Version 2013 puis les statistiques de base ont été réalisées avec le logiciel R Commander version 3.2.3. Dans l'interprétation des résultats, une différence n'a été considérée significative que si $\mathrm{p}<0,05$.

Le test ANOVA a été utilisé pour estimer les variations dues soit à l'âge, soit au sexe. Pour ce faire, les données sur l'âge ont été codées en deux grands groupes: adultes pour les animaux de plus de 3 ans et jeunes pour les moins de 3 ans.

Pour l'appréciation de la justesse et la fiabilité des différentes mesures, la répétabilité et la reproductibilité de la mesure des plis de peau ont été déterminées suivant la méthode de Charrette et al. (1996). Ces derniers définissent la répétabilité comme étant l'accord entre les mesures faites par les mêmes instruments, sur le même animal, par le même observateur sur une courte période. La reproductibilité se définit comme étant l'accord entre les mesures faites à l'aide du même instrument, par différents observateurs, sur le même animal sur une courte période. L'appréciation de ces deux paramètres par le modèle de Charrette et al. (1996) est fournie par l'équation suivante :

$\mathrm{Y}_{\mathrm{ijk}}=\mu \ldots+0_{\mathrm{i}}+\mathrm{A}_{\mathrm{j}}+\mathrm{OA}_{\mathrm{ij}}+\mathrm{Rk}(\mathrm{i})+\mathrm{e}_{\mathrm{ijk}}$

avec

$\mathrm{Y}_{\mathrm{ijk}}$ : individu,

$\mu$ : moyenne,

$0_{i}$ : effet de l'observateur $(i=1-3)$,

$A_{j}$ : effet de l'animal $(j=1-10)$,

$\mathrm{OA}_{\mathrm{ij}}$ : interaction entre l'effet de l'animal et de l'observateur,
$\mathrm{Rk}(\mathrm{i})$ : réplication de l'observateur $(\mathrm{k}=1-10)$, $\mathrm{e}_{\mathrm{ijk}}$ : erreur.

Les variances $\left(\mathrm{s}^{2}\right)$ des composantes du modèle ont été déterminées grâce au procédé VARCOMP du logiciel SAS version 9. A partir de ces variances, la répétabilité $\left(\mathrm{r}^{1}\right)$ et la reproductibilité $\left(\mathrm{r}^{2}\right)$ ont été calculées en utilisant les formules suivantes :

$\mathrm{r}^{1}=\left(\mathrm{s}^{2} \mathrm{~A}+\mathrm{s}^{2} 0+\mathrm{s}^{2} \mathrm{OA}\right) / \mathrm{s}^{2}$ total

$\mathrm{r}^{2}=\mathrm{s}^{2} \mathrm{~A} / \mathrm{s}^{2}$ total

Avec $\mathrm{s}^{2}$ total $=\mathrm{s}^{2} \mathrm{~A}+\mathrm{s}^{2} 0+\mathrm{s}^{2} 0 \mathrm{~A}+\mathrm{s}^{2} \mathrm{R}+$ $s^{2}$ erreur.

\section{RESULTATS}

Trois cents (300) mesures ont été effectuées, $120 \mathrm{chez}$ les adultes et $180 \mathrm{chez}$ les jeunes. L'âge moyen des animaux était de 4,5 ans [0,8-9 ans (IC 95\%)] et les mesures moyennes des plis de peau étaient de $9,14 \mathrm{~mm}$ [2,09-25,62 $\mathrm{mm}$ (IC 95\%)] pour la face droite et $9,7 \mathrm{~mm}[3,67-15,92 \mathrm{~mm}$ (IC $95 \%$ )] pour la face gauche. Les résultats montrent que les femelles Gobra ont une peau plus fine que celle des mâles (Tableau 1).

\section{Analyse de variance}

Cent cinquante (150) mesures tant chez les femelles que les mâles, ainsi que $120 \mathrm{chez}$ les adultes et 180 chez les jeunes ont été testés à l'analyse de variance. L'analyse de variance (Tableau 2) révèle que quel que soit la face, la variation des mesures de pli de peau est liée à l'âge et au sexe des animaux. La tendance est que l'épaisseur du pli de peau est beaucoup plus grande chez les mâles et les adultes. Par contre, il n'y a pas de différence significative entre les mesures d'une face à l'autre.

\section{Répétabilité et reproductibilité}

Les mesures du côté gauche ont été plus uniformes et avec moins de variations $(90,14 \%)$ et plus reproductibles $(86,66 \%)$ que celles de la face droite (Tableau 2).

La variabilité et la validité des mesures font l'objet de notre discussion. 


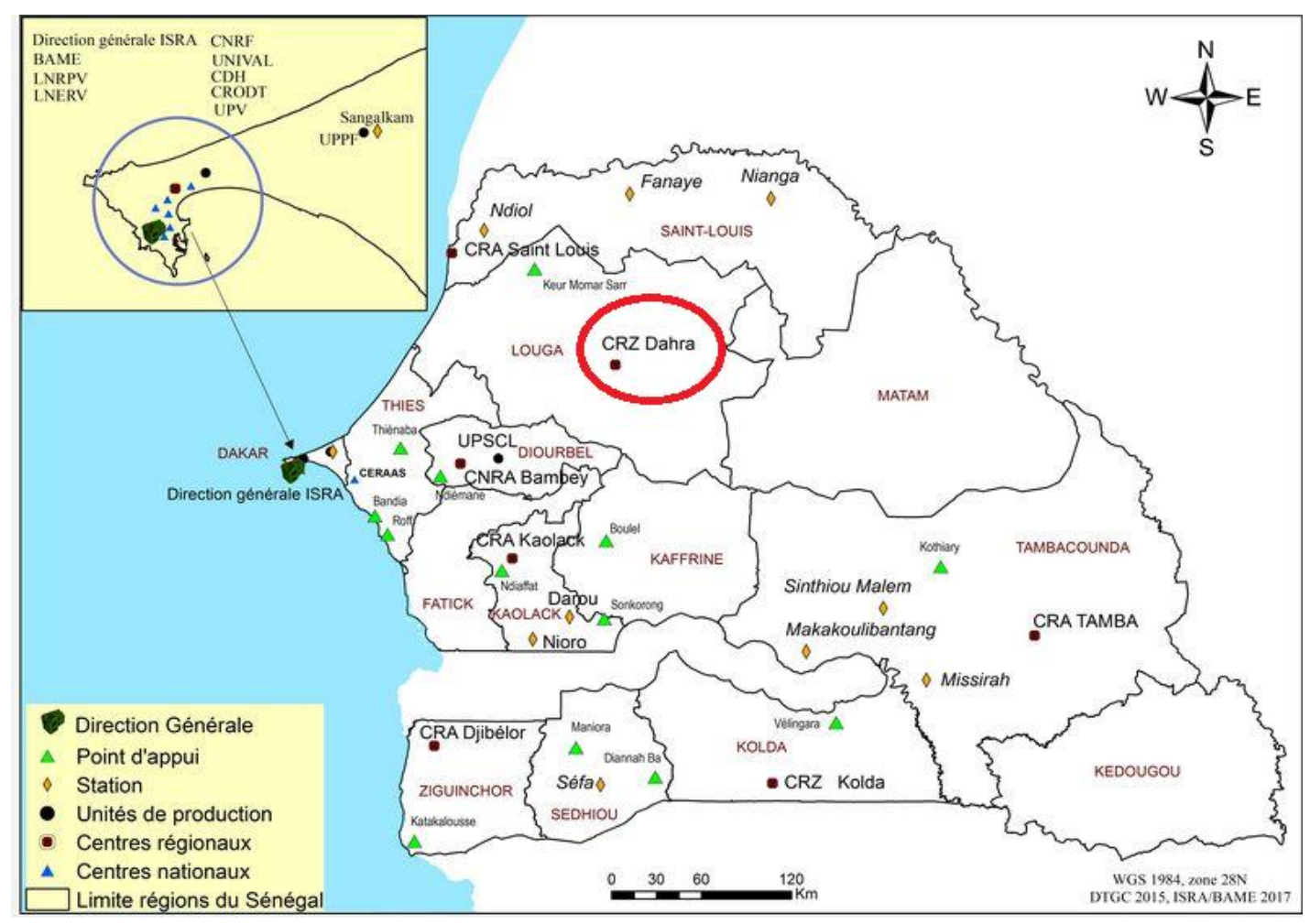

Figure 2 : Localisation du CRZ de Dahra Djoloff (www.isra.sn).

Tableau 1 : Moyennes des mesures du pli cutané (en mm) en fonction du sexe des bovins, Senegal, en 2016.

\begin{tabular}{llllll}
\hline & & Droit & \multicolumn{3}{c}{ Gauche } \\
\hline & Moyenne & IC $\mathbf{( 9 5 \% )}$ & Moyenne $(\mathbf{m m})$ & IC (95\%) \\
\hline Sexe & Femelle & 7,31 & $3,67-15,92$ & 7,9 & $4,6-16,5$ \\
& Mâle & 12,12 & $2,09-25,62$ & 11,66 & $3,67-24,37$ \\
\hline
\end{tabular}

Tableau 2 : Test ANOVA sur les mesures de pli de peau (en mm), zébu Gobra, par rapport à l'âge et au sexe en 2016 .

\begin{tabular}{|c|c|c|c|c|c|c|c|c|}
\hline & & $\mathbf{n}$ & & Moyenne & ET & SC & $\mathbf{F}$ & $\mathbf{p}$ \\
\hline & \multicolumn{8}{|c|}{ IC $(95 \%)$} \\
\hline & \multicolumn{8}{|c|}{ MESURES DROITES } \\
\hline \multirow[t]{2}{*}{ Age } & Adulte & 120 & 13,345 & $12,442-14,248$ & 5,050 & 1743,8 & 119,96 & $<2,2 \mathrm{e}^{-16^{*}}$ \\
\hline & Jeune & 180 & 8,424 & $8,013-8,817$ & 2,691 & & & \\
\hline \multirow[t]{3}{*}{ Sexe } & Femelle & 150 & 8,244 & $7,833-8,655$ & 2,571 & 1384 & 87,906 & $<2,2 \mathrm{e}^{-16^{*}}$ \\
\hline & Mâle & 150 & 12,540 & $11,742-13,338$ & 4,987 & & & \\
\hline & \multicolumn{8}{|c|}{ MESURES GAUCHES } \\
\hline \multirow[t]{2}{*}{ Age } & Adulte & 120 & 13,482 & $12,656-14,308$ & 4,617 & 1746,2 & 147,97 & $<2,2 \mathrm{e}^{-16^{*}}$ \\
\hline & Jeune & 180 & 8,557 & $8,216-8,898$ & 2,339 & & & \\
\hline
\end{tabular}




\begin{tabular}{ccccccccc}
\hline Sexe & Femelle & 150 & 8,758 & $8,318-9,198$ & 2,752 & 938,6 & 64,681 & $2,10 \mathrm{e}^{-14^{*}}$ \\
& Mâle & 150 & 12,296 & $11,555-13,037$ & 4,631 & & & \\
\hline
\end{tabular}

Légende

$\mathrm{SC}=$ Somme des carrés

$\mathrm{ET}=$ Ecart-type

$*=\mathrm{p}$ significatif $(\mathrm{p}<0,05)$

$\mathbf{F}=$ Valeur $\mathrm{F}$ de l'ANOVA

Tableau 3 : Répétabilité et reproductibilité des mesures de plis de peau chez le zébu Gobra (n=10 observateurs) Sénégal, 2016.

\begin{tabular}{lll}
\hline Paramètres & Mesure droite & Mesure gauche \\
\hline Variance liée à l'observateur & 0,51609 & 0,25882 \\
Variance liée à l'animal & 18,43365 & 16,77559 \\
Variance liée à l'animal x l'observateur & 0,47273 & 0,41492 \\
Variance liée à la réplication & 0,63054 & 0,31251 \\
Erreur & 2,30561 & 1,59544 \\
\hline$S^{2}$ total & 22,35322 & 19,35728 \\
Répétabilité (r1) & 0,86864756 & 0,90143502 \\
Reproductibilité (r2) & 0,824653 & 0,86662951 \\
\hline
\end{tabular}

\section{DISCUSSION}

Très peu d'études ont été consacrées à la mesure de l'épaisseur du pli de peau aussi bien dans les pays européens qu'en Afrique. Cependant, la différence d'épaisseur de peau entre les espèces Bos taurus et Bos indicus est rapportée depuis longtemps (Jian et al., 2013).

\section{Variabilité des mesures}

Les niveaux élevés de reproductibilité et de répétabilité, quelle que soit la face, seraient liés à l'animal, à l'instrument de mesure ou encore à l'opérateur. La sensibilité à la tuberculine est, en effet, influencée par la nature et la quantité de tuberculine administrée, le temps de lecture après injection, le statut immunologique de l'animal, mais, également de l'opérateur (de la Rua-Domenech et al., 2006). Une répétabilité de $84 \%$ et une reproductibilité de $64 \%$ ont été suffisantes pour valider le protocole d'évaluation de la note d'état corporel des porcs métis (Large White $\mathrm{x}$ race locale) au Sénégal (Manishimwe et Koné, 2015) par la méthode de Charrette et al. (1996), d'où l'adoption de la même approche méthodologique pour cette investigation. $\mathrm{La}$ détermination de ces deux paramètres est un moyen d'apprécier le niveau de précision de la méthode mais de vérifier, également, l'existence d'une certaine constance dans les résultats en fonction du temps (Arnal, 2017).

En France, les données issues d'une étude sur des troupeaux sentinelles donnent une épaisseur moyenne du pli de peau de 0,7 $\mathrm{mm}$ pour les allaitantes et de $0,3 \mathrm{~mm}$ pour les laitières avec des écarts-type respectifs de 1 et 0,6 $\mathrm{mm}$ (Lambert et Bénet, 2016). En comparant ces résultats aux nôtres, on se rend compte que la peau de Bos indicus est plus épaisse que celle de Bos taurus, ce qui a déjà été rapporté par Jian et al. (2013). De même, dans cette investigation, la tendance est que la peau soit beaucoup plus épaisse chez les 
mâles que chez les femelles, idem chez les adultes comparés aux jeunes. Ceci s'expliquerait par le fait que la structure de la peau des bovins est dépendante de l'âge, du sexe, ainsi que des aspects anatomiques et génétiques (Jian et al., 2013).

Habituellement, la mesure du pli de peau lors de la tuberculination par la voie intradermique chez les bovins se fait à l'aide d'un pied à coulisse traditionnel offrant une précision de 0,1 à $0,2 \mathrm{~mm}$. La lecture se fait lors d'une observation visuelle, à la fois, sur le vernier et sur la règle. L'utilisation d'un tel outil nécessite donc une certaine maitrise technique, contrairement, au cutimètre digital. Le cutimètre digital électronique utilisé offre une précision de 0,001/0,02 $\mathrm{mm}$, ce qui a justifié son adoption. Son utilisation a permis de réduire notoirement les variabilités à la lecture des résultats, la mesure étant lue sur le cadran digital de l'appareil.

Le nombre de manipulateurs et leur dextérité, la fréquence des mesures sont autant de paramètres pouvant influencer la reproductibilité et la répétabilité des mesures. Plus le nombre d'opérateurs et/ou de mesures est important, plus précis et justes seront les résultats qu'importe la variation entre individus (Arnal, 2017). Dans l'étude sur le porc métis au Sénégal, les mesures ont été répétées à trois reprises sur le même animal par cinq manipulateurs, soit un total de 150 mesures, pour une expérimentation menée sur 10 animaux (Manishimwe et Koné, 2015). Au cours de notre étude, le nombre de répétitions par animal a été le double de celui de Manishimwe et Koné (2015) d'où les niveaux élevés de fiabilité et de précision que nous avons obtenus. Il a été en outre constaté que les opérateurs étant tous droitiers, il leur était plus facile de faire le pli de peau sur la face droite de l'encolure que sur la gauche. Lors de la contention des animaux, la tête de l'animal est généralement déviée du côté droit, ce qui rend les muscles de la face gauche beaucoup plus tendus. Les manipulateurs n'ont pas la même facilité pour réaliser le pli de peau sur cette face gauche comparée à la droite ce qui justifierait le fait que les mesures de la face gauche soient plus élevées que celles de la face droite. Cette différence serait plutôt due à la manipulation qu'à la structure intrinsèque de la peau.

\section{Validation du protocole alternatif}

Les standards en matière

d'interprétation des tests en santé animale sont, le plus souvent, établis suivant des standards ne tenant pas compte des paramètres de variabilité géographique et d'espèce par exemple et, la tuberculination n'y a pas fait exception. En effet, dans l'interprétation des résultats de l'hypersensibilité retardée, les seuils de détection (OIE, 2012) en fonction de l'accroissement du pli de peau (positif si $>4$ $\mathrm{mm}$, négatif si $<2 \mathrm{~mm}$, douteux si compris entre 2-4 mm) ont été fixés dans un contexte de faibles prévalence et incidence de la tuberculose, pour une espèce donnée, Bos taurus. Compte tenu des différences de peau pouvant exister en fonction de la race, du sexe, de la zone géographique et du contexte épidémiologique (de la Rua-Domenech et al., 2006), il est possible de classer négatif un animal pourtant porteur du germe d'où la nécessité de définir des seuils propres au contexte africain. Le dépistage de la TbB par la tuberculination est très peu pratiqué en Afrique et; seuls $10 \%$ des bovins seraient régulièrement dépistés en Afrique subsaharienne (Boukary et al., 2011). Au cours de nos travaux, le constat a été que très peu de vétérinaires maîtrisent la technique de tuberculination; un renforcement de leurs capacités s'avère donc nécessaire. De même, vu les niveaux élevés de fiabilité ( $>90 \%$ ) et de précision $(>80 \%)$ du pli cutané obtenus lors de notre expérimentation, on pourrait alors envisager la possibilité d'effectuer la mesure à $\mathrm{J} 0$ en point symétrique lors du deuxième passage (soixante-douze après l'injection de la tuberculine) si, toutefois celle-ci n'a pas été effectuée avant l'injection de la tuberculine. Cependant, cela n'exclue pas l'existence de deux visites dans le troupeau, une première pour injecter la tuberculine sans faire la 
mesure du pli de peau et la seconde, soixantedouze heures plus tard. C'est lors de cette dernière que les deux mesures du pli de peau sur chacune des faces de l'encolure devront être faites au cours de la même contention de l'animal (contention n'excédant pas deux minutes). L'intérêt est donc le gain de temps substantiel lors de l'immobilisation des animaux. Egalement, lors de la tuberculination, cette approche permettrait de tuberculiner un nombre beaucoup plus conséquent d'animaux que l'approche classique.

\section{Conclusion}

Notre étude sur l'appréciation de la fiabilité et de la précision des mesures du pli de peau est la première du genre chez le zébu Gobra au Sénégal. Elle cadre parfaitement avec les objectifs mondiaux d'éradication de la tuberculose se rapportant à la nécessité de l'amélioration des connaissances scientifiques sur la pathologie, à la réduction de la transmission à l'interface homme-animal et, au développement de partenariats intégrés multisectoriels.

La possibilité de faire la mesure du pli de peau du Jour 0 au Jour 3 en un point symétrique sur la face opposée de l'encolure en même temps que le résultat de la tuberculination a été envisagée afin d'évaluer dans quelle mesure elle peut être avantageuse plus particulièrement lors de tuberculination comparative. A l'issue des travaux, il s'avère que les mesures au niveau de la face gauche (reproductibles à $90,14 \%$ et répétables à $86,66 \%$ ) étaient beaucoup plus précises et fiables que celles de la face droite (reproductibles à $86,86 \%$ et répétables à $82,46 \%)$. De même pour des sujets de même sexe et/ou de même âge, aucune différence significative dans l'épaisseur du pli de peau n'a été constatée. Faire la mesure de J0 soixante-douze heures (J3) après inoculation de la tuberculine en un point symétrique de l'encolure pourrait donc être envisagée chez le zébu Gobra, Bos indicus Linnaeus, 1758. Néanmoins, les expérimentations doivent être poursuivies pour apprécier sa concordance réelle avec la méthode standard de l'OIE et son utilisation dans un milieu d'enzootie de tuberculose bovine.

\section{CONFLIT D'INTERETS}

Les auteurs déclarent qu'ils n'ont aucun conflit d'intérêts.

\section{AUTHORS' CONTRIBUTIONS}

Les auteurs ont contribué aussi bien à la réalisation des travaux qu'à la rédaction du document.

\section{REMERCIEMENTS}

Les auteurs adressent leurs sincères remerciements aux autorités et aux stagiaires du Centre de Recherches Zootechniques de Dahra Djoloff pour leur implication et leur partenariat dans la réalisation de cette investigation.

\section{REFERENCES}

Alvarez J, Perez A, Bezos J, Marques S, Grau A, Saes JL, Minguez O, De Juan L, Domingo L. 2012. Evaluation of the sensitivity and specificity of bovine tuberculosis diagnostic tests in naturally infected cattle herds using a Bayesian approach. Veterinary Microbiology, 155: 38-43.

DOI: 10.1016/j.vetmic.2011.07.034

Amanfu W. 2006. The situation of tuberculosis and tuberculosis control in animals of economic interest. Tuberculosis, 86 : 330-335. DOI : 10.1016/j.tube.2006.01.007

Arnal F. 2017. Répétabilité et reproductibilité d'une méthode. Polycopié de cours. Université de Bordeaux, 11P.

Ayele WY, Neill SD, Zinsstag J, Weiss MG, Pavlik I. 2004. Bovine tuberculosis: an old disease but a new threat to Africa. Int J Tuberc Lung Dis, 8(8): 924-937.

Boukary AR, Thys E, Mamadou S, Rigouts L, Matthys F, Vias Franck SG, Gamatie D, Yenikoye A, Saegerman C. 2011. La tuberculose à Mycobacterium bovis en Afrique subsaharienne. Ann. Méd. Vét., 155 : 23-37. 
Chambers MA. 2013. Review of the Diagnosis of Tuberculosis in Non-Bovid Wildlife Species Using Immunological Methods - An Update of Published Work Since 2009. Transboundary and Emerging Diseases, 60(1): 14-27. DOI: https://doi.org/10.1111/tbed.12094

Charrette B, Bigras-Poulin M, Martineau G-P. 1996. Body condition evaluation in cow. Livestock Production Science: 107-115.

De Garine-Wichatitsky M, Caron A, Kock R, Tschopp R, Munyeme M, Hofmeyr M, Michel A. 2013. A review of bovine tuberculosis at the wildlife-livestockhuman interface in sub-Saharan Africa. Epidemiol. Infect., p. 15. DOI: 10.1017/S0950268813000708

De la Rua-Domenech R, Goodchild AT, Vordermeier HM, Hewinson RG, Christiansen KH, Clifton-Hadley RS. 2006a. Ante mortem diagnosis of tuberculosis in cattle: A review of the tuberculin test, $\gamma$-intereferon assay and other acillary diagnostic techniques. Research in Veterinary Science, 81: 190-210

Diagne ST. 2009. Tuberculose bovine aux abattoirs de Dakar_souches de Mycobactéries sur 200101 carcasses inspectées de 2005-2008. Thes. Med. Vet, $\mathrm{n}^{\circ} 22: 130 \mathrm{p}$.

Jian W, Duangjinda M, Vajrabukka C, Katawatin S. 2013. Differences of skin morphology in Bos indicus, Bos taurus, and their crossbreds. Int J Biometeorol. DOI: 10.1007/s00484-013-0700-9

Kwaghe AV, Ameh AJ, Ambali A-G, Kudi AC, Kachalla MG. 2015. Prevalence and Economic Losses from Bovine Tuberculosis in Maiduguri, Borno State, Nigeria. International Journal of Life Sciences, 4(4) : 283-287.

Lambert O, Benet JJ. 2016. Evaluation d'un protocole alternatif de mesure du pli de peau lors de la tuberculination chez les bovins. Bulletin Epidemiol. Santé Animale \& Alimentaire, 73 : 14-15.

Manishimwe R, Kone P. 2015. Mise en place d'une méthode d'évaluation de l'état corporel du porc métis (Large White et Race Locale) au Sénégal. Epidemiol. et Santé animale, 68(4): 95-104.

OIE. 2012. Bovine tuberculosis. Manual of Diagnostic Tests and Vaccines for Terrestrial Animals (7th Edn). OIE; 674689.

OMS, OIE, FAO, L'Union. 2017. Feuille de route pour la tuberculose zoonotique, $\mathrm{p}$. 20.

Schiller I, Oesch B, Vordermeier HM, Palmer MV, Harris BN, Orloski KA, Buddle BM, Thacker TC, Lyashchenko KP, Waters WR. 2010. Bovine tuberculosis: a review of current and emerging diagnostic techniques in view of their relevance for disease control and eradication. Transboundary and Emerging Diseases, 57 : 205-220. DOI : 10.1111/j.1865-1682.2010.01148.x

Sénégal. Ministère de l'Elevage et des Productions Animales. 2015. Rapports d'activités, $70 \mathrm{p}$.

Vikou R, Aplogan LG, Ahanhanzo C, BabaMoussa L, Gbangboche B. 2018. Prévalence de la brucellose et de la tuberculose chez les bovins au Bénin. Int. J. Biol. Chem. Sci., 12(1): 120-128. DOI:

https://dx.doi.org/10.4314/ijbcs.v12i1.9

Zinsstag J, Kazwala RR, Cadmus I, Ayanwale L. 2006. Mycobacterium bovis in Africa. In Mycobacterium bovis Infection in Animals and Humans (2nd edn), Thoen CO, Steele JH, Gilsdorf MF (eds). Blackwell Publishing Professional: Ames, Iowa, USA; 199-210. 